\title{
Viscous effects on the position of cavitation separation from smooth bodies
}

\author{
By VIJAY H. ARAKERI† \\ California Institute of Technology, Pasadena
}

(Received 9 April 1973 and in revised form 31 May 1974)

Flow visualization by the schlieren technique in the neighbourhood of a fully developed cavity on two axisymmetric headforms has shown the existence of laminar boundary-layer separation upstream of cavitation separation, and the distance between the two separations to be strongly dependent on Reynolds number. Based on present results, a semi-empirical method is developed to predict the position of cavitation separation on a smooth body. The method applies only in the Reynolds-number range when the cavitating body possesses laminar boundary-layer separation under non-cavitating conditions. Calculated positions of cavitation separation on a sphere by the method show good agreement with experimentally observed positions.

\section{Introduction}

Following Plesset (1949), one may divide the flow of a liquid around a solid body into three regimes. (i) In non-cavitating flow, the liquid follows the classical hydrodynamic laws for an incompressible fluid. (ii) In what may be called limited or incipient cavitation, small discrete bubbles or areas occupied by patches of gas or vapour first appear. (iii) Fully cavitating or super-cavitating flow is characterized by a single large vapour or gas cavity enveloping some portion of the body. Super-cavitating flow is of primary concern here. In particular, we are interested in viscous effects on the beginning position of the developed cavity, which we may call the position of cavitation separation.

To predict analytically the force coefficients on bodies with a fully developed cavity, knowledge of the position of cavitation separation is essential. Bodies with sharp corners (such as disks, wedges and sharp-edged hydrofoils at moderately large angles of attack) possess a cavitation separation point whose position is known a priori; and force coefficients for such bodies can be predicted quite well within the potential flow approximations ( $\mathrm{Wu} 1968$ ). But, for smooth bodies, the condition of smooth separation is usually used to predict the position of cavitation separation. The classical condition for smooth separation requires that the curvature of the free streamlines be finite at separation, in which case it can be shown to be equal to the curvature of the solid body at the detachment point (Wu 1968; Armstrong 1953). This condition does not take into account real fluid effects afforded by viscosity, surface tension, etc., on the position of cavitation separation. Recent experimental studies (Brennen 1969a, $b$; Arakeri 1971) have shown that the position of cavitation separation on smooth bodies at least $\dagger$ Present address: Energy and Kineties Department, University of California, Los Angeles. 
depends on Reynolds number. Furthermore, it was also observed that the experimentally found position of cavitation separation lies considerably downstream from that predicted by the condition of smooth separation. On a sphere (Brennen $1969 a$ ), for example, the difference was of the order of $40^{\circ}$ at a Reynolds number of $10^{4}$; and the difference decreased with increase in Reynolds number. Similar results were noted for a bi-convex hydrofoil (Arakeri 1971). On this hydrofoil, linearized theory with eavitation separation positions determined by the smooth-separation condition predicted positive lift coefficients at small positive angles of attack. But experiment showed negative lift coefficients at the ame angles of attack. This discrepancy was directly related to the erroneous prediction of cavitation separation position by the smooth-separation criterion. This latter example illustrates the extremely important role the position of the point of cavitation separation has in determining overall forces.

This study went further (Arakeri 1971), to describe basically two different types of cavitation separation; these are termed (i) viscous laminar and (ii) nucleate. The physical appearance of (i) is very different from that of (ii); type (i) possesses a smooth glassy surface cavity at the position of separation. Type (ii) exhibits large macroscopic cavitation bubbles at the position of separation. Figure 1 (plate 1) clearly illustrates the difference. From Arakeri (1973) we have a better idea of what is physically involved in at least one type of cavitation separation. It may be said that viscous laminar cavitation separation is observed only on bodies which possess a laminar boundary-layer separation under non-cavitating conditions at the same Reynolds number as that existing under cavitating conditions. It is this type of cavitation separation whose position is predicted quite inaccurately using the smooth-separation condition. By comparison, the position of nucleate cavitating separation is predicted quite accurately using the same condition (Arakeri 1971).

The present work examines the nature of real fluid flow in the neighbourhood of viscous laminar cavitation separation, with the schlieren technique as a flow visualization method. Based on data from these observations, a semi-empirical method of predicting the position of viscous laminar cavitation separation on an arbitrary smooth body is proposed, which is intended to replace that of the pure mathematically inferred smooth-separation condition.

\section{Experimental methods}

Conventional methods of flow visualization (such as dye injection and oil film techniques, and even the more recent hydrogen bubble procedure) are difficult to use in water at high velocities. For this reason, the well-known schlieren technique of flow visualization, widely used in compressible aerodynamics applications, was used during the present experiments. The schlieren method was shown to work in water by Bland \& Pelick (1962) and Arakeri (1973). The density gradients necessary for the method were created by heating or cooling of the test body. In the present experiments the schlieren method of flow visualization was primarily used to detect the existence of laminar separation of the viscous boundary layer. This may be adequately accomplished by 
observing the thermal boundary layer created by heating or cooling of the test body. But the fact that the Prandtl number of water at room temperatures is close to 7 does remove the possibility of obtaining quantitative information on the extent of the viscous or vorticity boundary layer by the schlieren method. As mentioned later, the viscous boundary-layer growth on the present test bodies was calculated by Thwaites's (1949) method. Details of the optical apparatus are provided elsewhere (Arakeri 1973), but perhaps it should be noted here that a temperature difference of only $3-4{ }^{\circ} \mathrm{F}$ is sufficient to produce a clear thermal boundary layer. Within such a range of temperature differences, both heating and cooling were shown to produce equivalent effects. In the work to be discussed, heating was used, the source of the heat being a $1 \mathrm{~kW}$ electrical heater immersed within the test bodies.

The tests to be described were carried out in the axisymmetric test section of the High Speed Water Tunnel (HSWT) of the Hydrodynamics Laboratory, California Institute of Technology (Knapp, Levy, O'Neill \& Brown 1948). Some preliminary tests with the schlieren technique were carried out in the Free Surface Water Tunnel (FSWT) of the same laboratory. Two cylindrical axisymmetric bodies with different-shaped nose sections (see figure 2) were used for the present experimental studies. The test body with a hemispherical nose section is commonly known as the hemispherical nose, and the test body with a modified ellipsoidal nose section as the Swedish headform. Pressure distributions under non-cavitating conditions on the two bodies were measured by Rouse \& McNown (1948). The bodies were mounted in the test section of the HSWT by a three-bladed sting support, which was about 7 diameters downstream of the model nose. The bodies occupied less than $2 \%$ of the through-flow area.

The free-stream velocity in the tunnel $U$ and cavitation number $\sigma$ were determined from tunnel static pressure measurements, made with mercury manometers. ( $\sigma$ is given by $\left(P_{t}-P_{C}\right) / \frac{1}{2} \rho U^{2}$, where $P_{t}$ is the free-stream tunnel static pressure, $\rho$ is the liquid density and $P_{C}$ is the cavity pressure. Presently we shall be dealing only with vapourous cavities; hence $P_{C}$ is very close to the value of the vapour pressure of the liquid, $P_{v}$.) These computations included corrections for the boundary-layer growth along the tunnel walls, as outlined by Acosta \& Hamaguchi (1967). From computations of Hoyt (1966), blockage effects are negligible in the present experiments; and they were not accounted for in the data reduction. The normal experimental procedure was to hold the tunnel velocity fixed at a constant value, and slowly reduce the tunnel pressure, until a natural cavity was formed on the test body. A silhouette schlieren photograph was taken with a spark source, having a duration of 5-10 $\mu \mathrm{s}$. This procedure was repeated for several tunnel pressures, and for tunnel velocities $25-60 \mathrm{ft}^{-1} \mathrm{~s}$. A 'calibration' photograph was made to establish true lengths.

\section{Fully-wetted flow observations}

The effectiveness of the schlieren technique is illustrated in figure 3(a) (plate 1), which shows the laminar boundary-layer separation and re-attachment of the free shear layer on a $2 \mathrm{in}$. hemispherical nose mounted in the FSWT at a Reynolds 


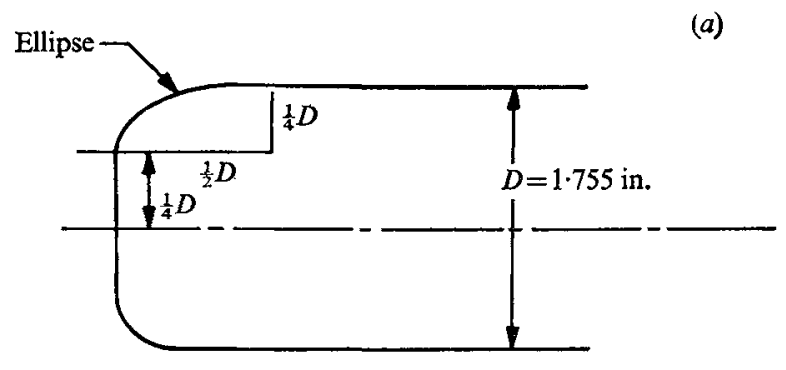

(b)

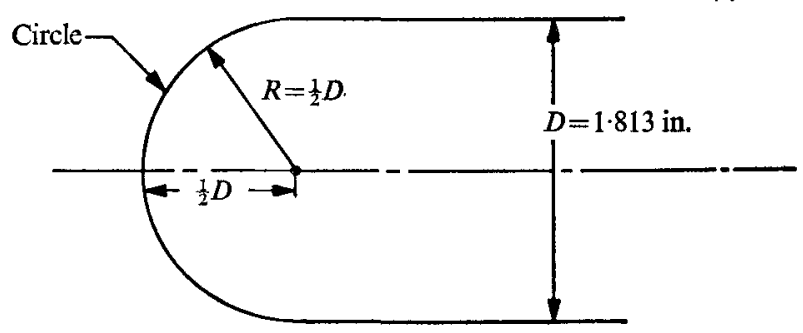

Frgure 2. Diagrams showing geometrical details of the axisymmetric models used.! (a) Swedish headform, a modified ellipsoidal nose. (b) Hemispherical nose.

number of $10^{5}(R=U D / \nu$ is the Reynolds number). The outer white line, which begins at the point marked 'position of laminar separation' in figure $3(a)$ could only be due to the presence of a sharp temperature gradient across a free shear layer, strongly indicating the existence of laminar separation. The presence of a reverse flow region at an $R$ of $10^{5}$ was confirmed by dye-injection studies, and by photographic study of the electrolysis bubbles created on the whole test body. Thus we are confident in interpreting the region shown by the outer white line in the schlieren photograph of figure $3(a)$ as a laminar separated region. From such observations it was found that both of the test bodies possessed a laminar separation 'bubble' at all the test velocities used in the HSWT. The highest test velocity corresponds to an $R$ of $9 \cdot 06 \times 10^{5}$ for the hemispherical nose, and $8.78 \times 10^{5}$ for the Swedish headform. Examples of schlieren photographs at higher Reynolds numbers are shown in figures $3(b),(c)$ (plate 1). The existence of a laminar separated region is strongly suggested by the photographs, and was confirmed by motion picture studies of electrolysis bubbles created on the test bodies. These studies (partly described by Arakeri 1973) showed the existence of reverse flow within what is interpreted as the separated region, from figures $3(b),(c)$. From the photographs of this type, the position of laminar separation was measured, and an estimate of the length of the separated region was made. The streamwise position of laminar separation $\left(S_{B} / D\right)_{\mathrm{NC}}, \dagger$ on the hemispherical nose (in both tunnels) and the Swedish headform, as a function of tunnel velocity,

$\uparrow$ The subscript NC denotes the quantity in the bracket existing under non-cavitating conditions. 


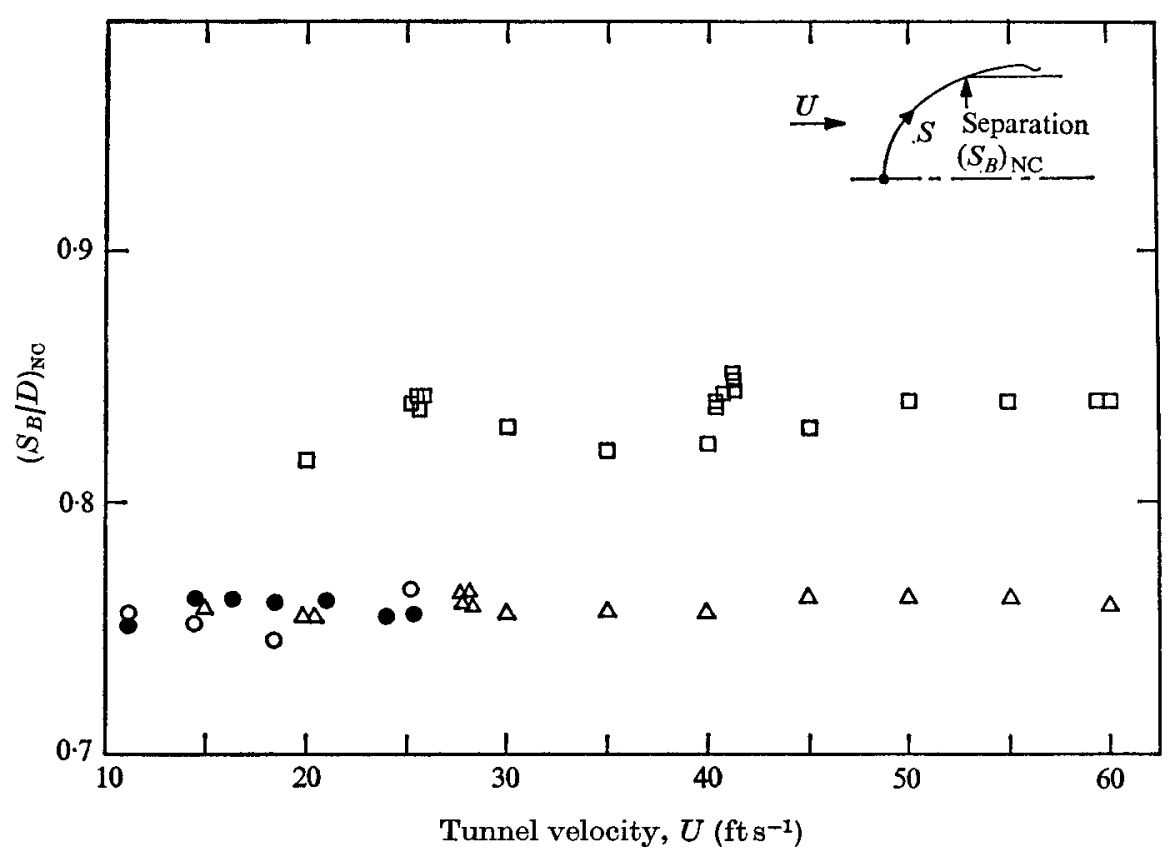

Figure 4. Measured streamwise position of laminar separation against tunnel velocity for the Swedish headform and two different hemispherical noses, under non-cavitating con. ditions. Also, effect of temperature on position.

\begin{tabular}{cllc}
\multicolumn{1}{c}{ Body } & \multicolumn{1}{c}{ Apparatus } & $D$ (in.) \\
$\square$ & Headform & HSWT & $\mathbf{1 \cdot 7 5 5}$ \\
$\triangle$ & Nose & HSWT & $\mathbf{1 \cdot 8 1 3}$ \\
0 & Nose & FSWT (heated) & $\mathbf{2 \cdot 0}$ \\
\hline & Nose & FSWT (cooled) & $\mathbf{2 \cdot 0}$
\end{tabular}

is shown in figure 4. From this figure, the position of laminar separation does not change appreciably with $U$, and is not altered by heating or cooling. This assured us that the boundary-layer characteristics on the test bodies used were not altered with the temperature differences used to create the schlieren effect.

In figures $3(b),(c)$ the outer white line, which indicates the boundary of the separated region, becomes wavy and subsequently too faint to be recorded. This is evidently the location of intense mixing signaling transition to turbulence; presumably re-attachment of the flow would soon follow. In any case, it seems reasonable that the representative length of the separated bubble is given by this distance. Variation of the distance so measured with tunnel velocity for both the test bodies is shown in figure 5. As expected, the bubble length $L_{r}$ decreases with increasing velocity, for both bodies. Physically these bubble lengths are quite small, being only $12 \%$ of the diameter at $20 \mathrm{ft} \mathrm{s}^{-1}\left(R \sim 3 \times 10^{5}\right)$ and about $4 \%$ at $60 \mathrm{ft} \mathrm{s}^{-1}\left(R \sim 9 \times 10^{5}\right)$. The length of separation bubble $L_{r}$, as defined earlier and normalized by the momentum thickness at separation $\theta_{s}$, is plotted in figure 6 as a function of local Reynolds number $R_{\theta_{s}}=U_{s} \theta_{s} / \nu$. Thwaites's (1949) method in addition to pressure distribution measurements of Rouse \& McNown (1948) were used to calculate $\theta_{s}$ and $R e_{\theta_{s}}$. Also shown in figure 6 are wind-tunnel data 


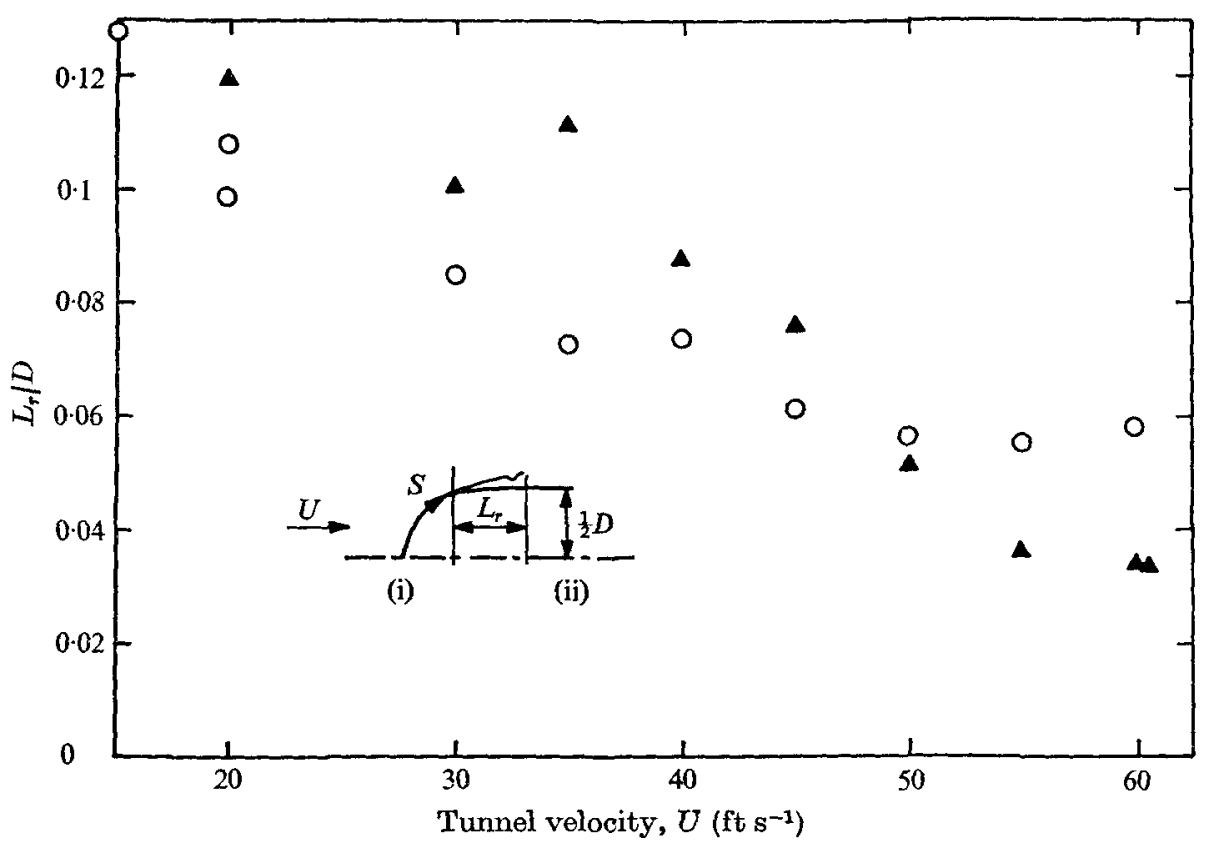

Figure 5. Length of laminar separated region against tunnel velocity for both test bodies. $\boldsymbol{\Delta}$, Swedish headform; $O$, hemispherical nose. (i) Position of boundary-layer separation; (ii) position of end of separated layer.

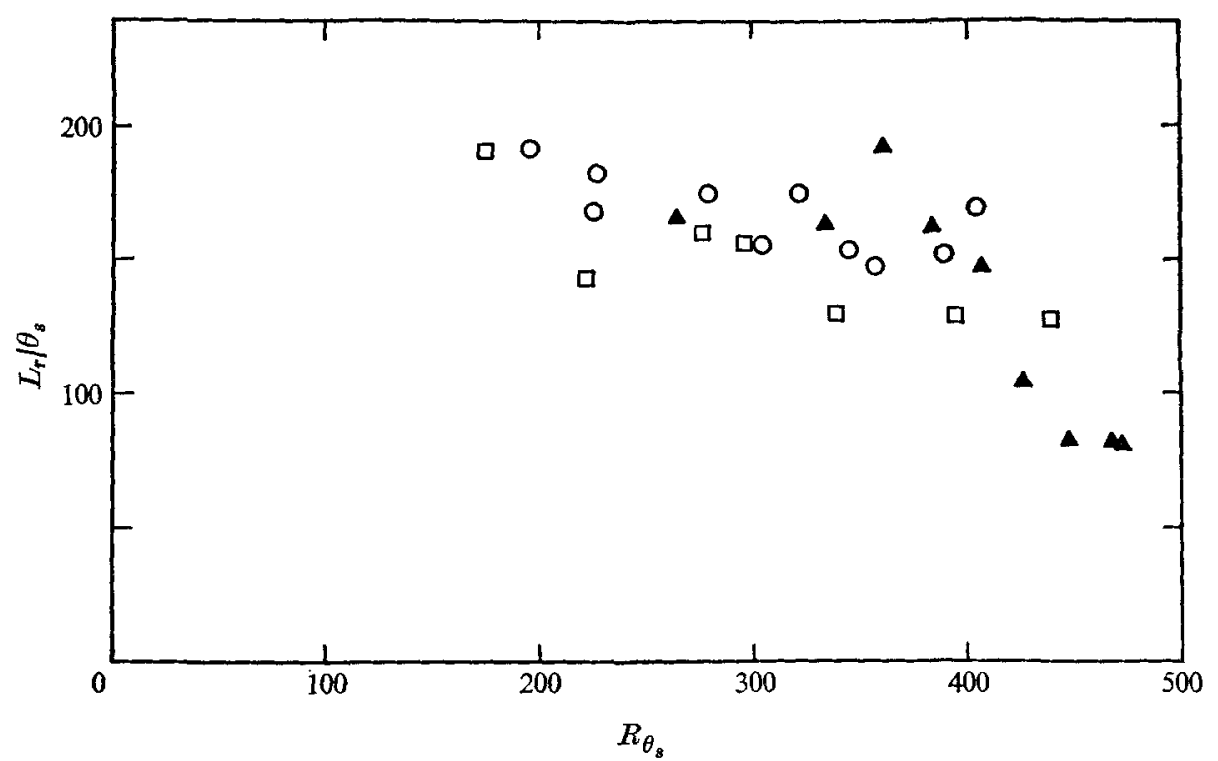

Figure 6. Comparison of our data on the length of the separated region ( $\Delta$, Swedish headform; $\bigcirc$, hemispherical nose) with wind-tunnel studies of Gaster ( $\square$ ). 
obtained by Gaster (1967) in his studies on laminar separation bubbles. A measure of the length of the separated bubble in his case was found from detailed velocity surveys. The separated region represented by this length is termed the dead-air region by Gaster. From figure 6 present findings agree well with Gaster's measurements.

\section{Flow observations with cavitation}

Cavitation on the test bodies was established by lowering the tunnel pressure or equivalently by lowering the cavitation number $\sigma$. The extent of cavitation on the hemispherical nose at different values of tunnel pressure is shown in the schlieren photographs of figure 7 (plate 2). Cavitation bubbles are first observed to originate within the reattachment portion of the separated region. With reduction in $\sigma$, a considerable portion of the separated region is observed to be filled by cavitation bubbles, and, with further reduction in $\sigma$, almost all of the separated region is occupied by a macroscopic attached smooth cavity. The beginning position of a smooth attached cavity in figure $7(c)$ is downstream of the position of laminar boundary-layer separation. This interpretation is further supported by the observations of Parkin \& Kermeen (1953). They photographically observed the reverse motion of microscopic cavitation bubbles $(\sim 0.002$ in. in diameter), originating on the test body surface immediately upstream of an attached smooth cavity on a hemispherical nose at speeds similar to those used in the present experiments. The reverse motion of microscopic bubbles also observed in the present work strongly suggests the existence of a recirculating flow region just upstream of attached cavitation. A schlieren photograph of the Swedish headform with a cavity considerably longer than that on the hemispherical nose observed in figure $7(c)$ is shown in figure 8 (plate 3 ). The outer white line away from the model surface upstream of cavitation separation indicates a free shear layer, which presumably reattaches to the cavity surface, to form a closed recirculating flow region. With the help of such photographs, it was possible to estimate the position of boundary-layer separation, the position of cavitation separation, and hence the distance $\lambda$ between the two.

Variation of the angular position of the cavitation separation $\gamma_{C}$ with $U$ and $\sigma$ for the hemispherical nose is shown in figure 9 . The position of cavitation separation shows a dependence on both $\sigma$ and $U$. Figure 10 shows the variation of the angular position of the boundary-layer separation $\gamma_{B}$, which precedes the cavitation separation on the hemispherical nose as a function of $U$ and $\sigma$. Unlike the position of cavitation separation, the position of the boundary-layer separation preceding it is independent of the tunnel velocity. Variation of the streamwise position of the cavitation separation $S_{C} / D$, and the boundary-layer separation $S_{B} / D$ preceding it, for the Swedish headform, as a function of $U$ and $\sigma$, are shown in figures 11 and 12. The results are similar to that for the hemispherical nose, discussed earlier.

The ratio of $\lambda$ to the diameter of the model $D$ is plotted against $\sigma$ for various tunnel velocities in figures $13(a),(b)$ (both test bodies). From these figures, $\lambda / D$ increases with decreasing $U$, but in general it is almost constant with change in 


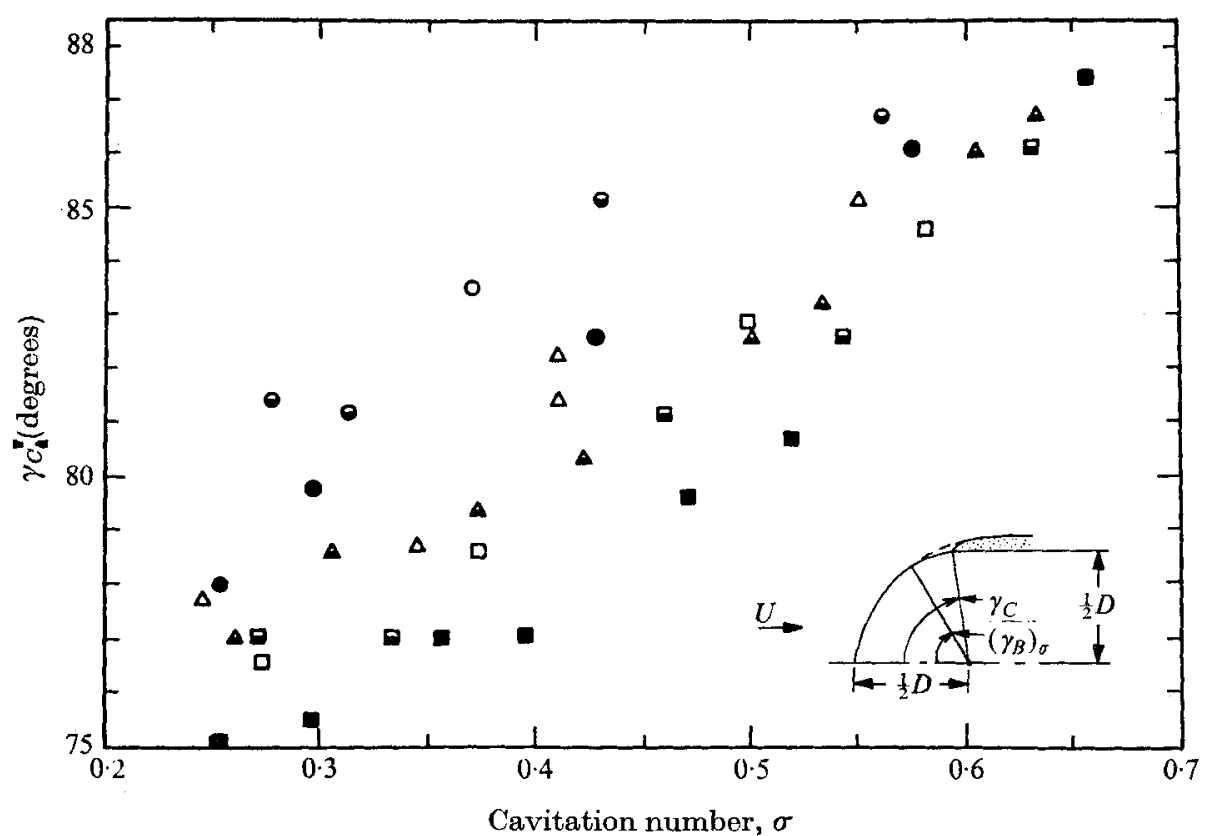

Figure 9. Variation of angular position of cavitation separation on the hemispherical nose with $\sigma$ and $U$.

$\begin{array}{lcrrrrrrr} & \bigcirc & \ominus & \bullet & \triangle & \Delta & \square & \square & \square \\ U\left(\mathrm{ft} \mathrm{s}^{-1}\right) & 20 & 25 & 30 & 35 & 40 & 45 & 50 & 55\end{array}$

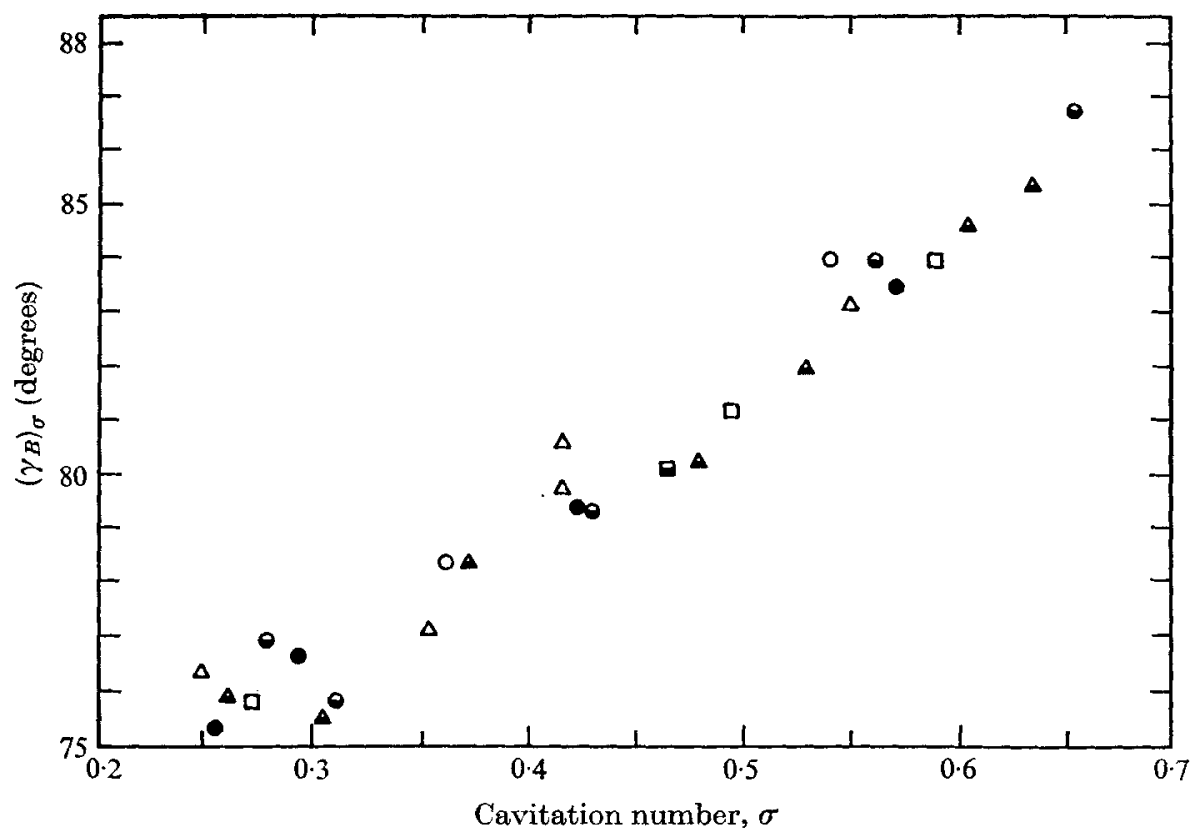

FraURe 10. Variation of angular position of boundary-layer separation, under cavitating conditions, on the hemispherical nose, with $\sigma$ and $U$. For symbols, see figure 9 . 


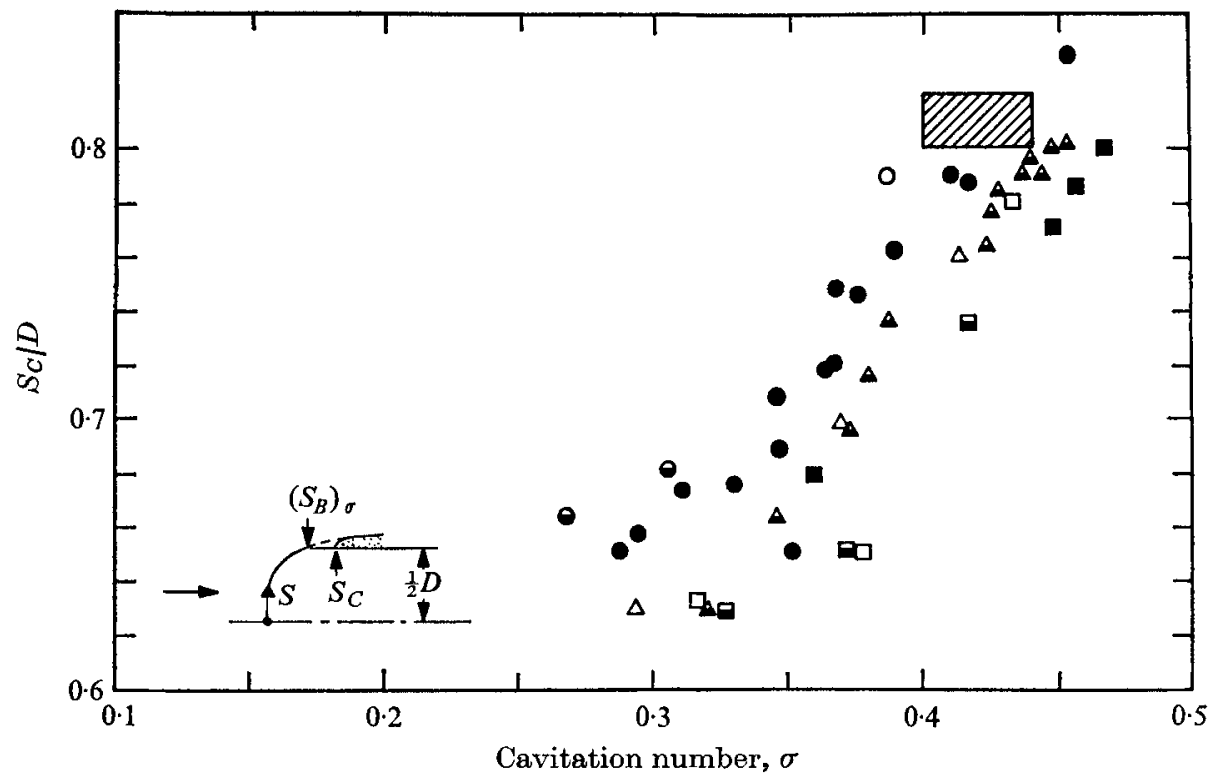

Figure 11. Variation of streamwise position of cavitation separation, on the Swedish headform, with $\sigma$ and $U$. For symbols, see figure 9. Acosta's results.

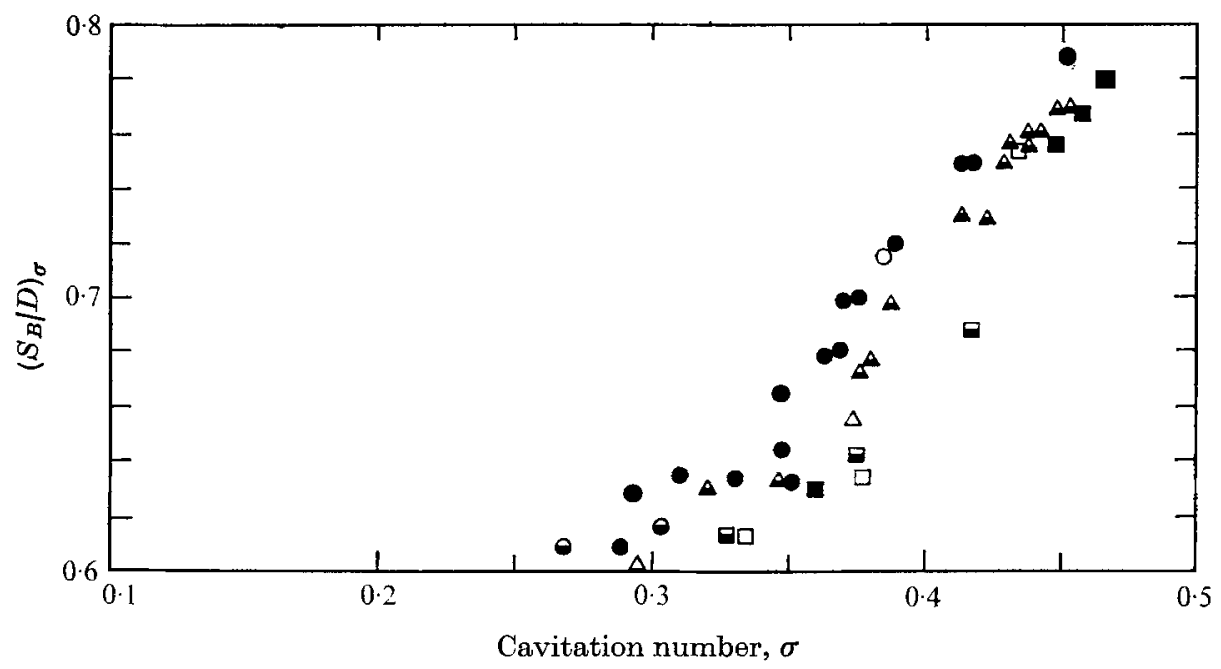

FIGURE 12. Variation of streamwise position of boundary-layer separation, under caritating conditions, on the Swedish headform, with $\sigma$ and $U$. For symbols, see figure 9 .

$\sigma$, or at least did not show any consistent dependence on $\sigma$ for a fixed velocity. Scatter at certain velocities is considerable and in general the values of $\lambda / D$ are quite small, the maximum being 0.07 and the minimum 0.01 . With the earlier observation of the independence of $\lambda / D$ and $\sigma$, it is convenient to take an average value of $\lambda / D$ for all the meaningful cavitation numbers: $\lambda^{*} / D$. This quantity is plotted against $U$ for both test bodies in figure 14. Each data point in figure 14 represents the average value of $\lambda / D$ along the horizontal axis of figure $13(a)$ or $(b)$, at the corresponding velocity. 

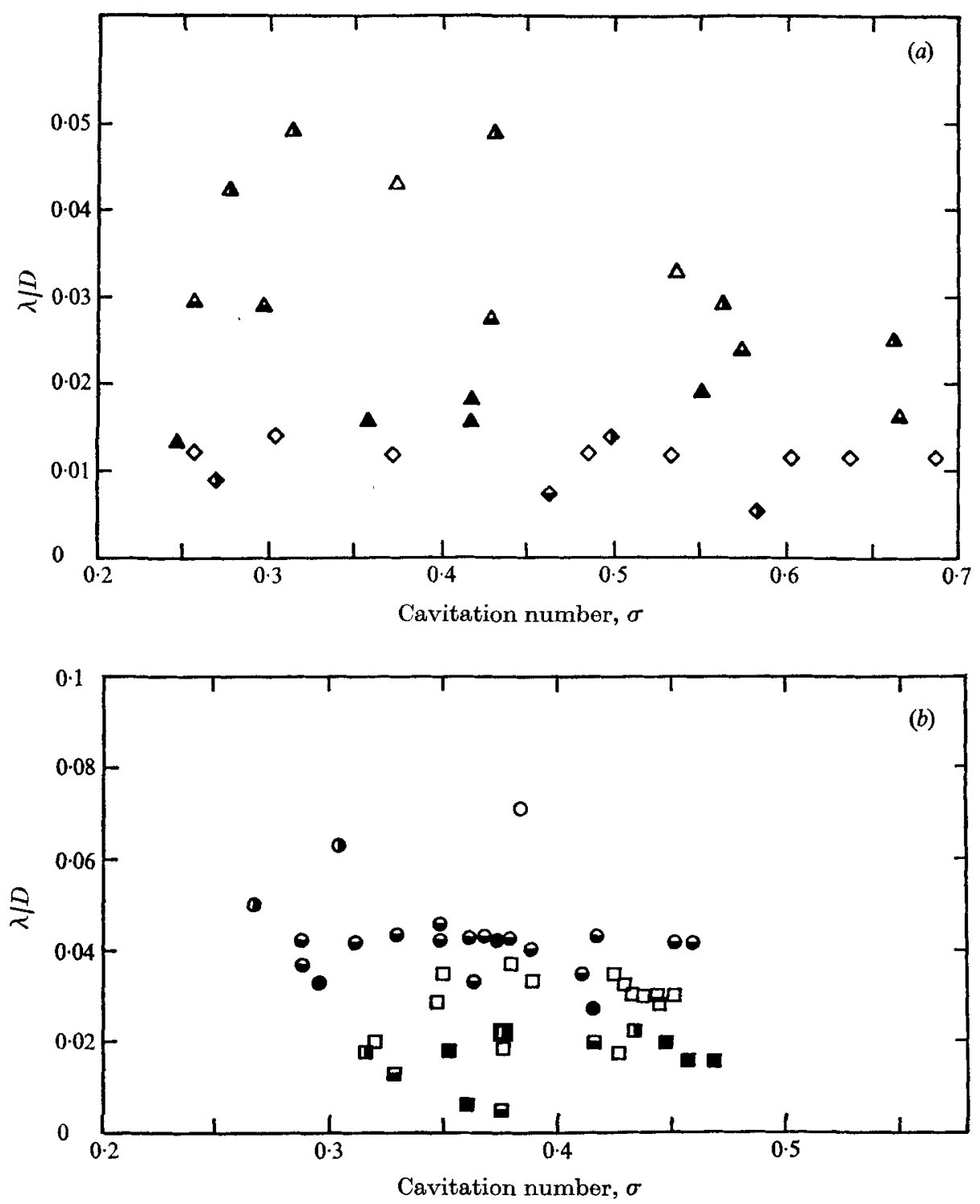

FIGURE 13. Variation of the streamwise distance between the two separations, with $\sigma$ and $U$, for $(a)$ hemispherical nose, $(b)$ Swedish headform.

\begin{tabular}{|c|c|c|c|c|c|c|c|}
\hline$U\left(\mathrm{ft}^{-1}\right)$ & 20 & 25 & 30 & 35 & 40 & 45 & 50 \\
\hline $\begin{array}{l}(a) \\
(b)\end{array}$ & $\triangle$ & $\underset{\mathbf{\Delta}}{\boldsymbol{\Delta}}$ & $\frac{\Delta}{\ominus}$ & 9 & $\stackrel{\diamond}{\square}$ & $\stackrel{\bullet}{\square}$ & $\stackrel{\vec{\theta}}{\square}$ \\
\hline
\end{tabular}

Now one can determine the position of cavitation separation once the position of the boundary-layer separation preceding it and the quantity $\lambda$ are known. On the present test bodies, observations strongly suggest that the position of the boundary-layer separation preceding the cavity separation is dependent only on $\sigma$, but is independent of $U$; and the quantity $\lambda$ is only dependent on $U$, but 


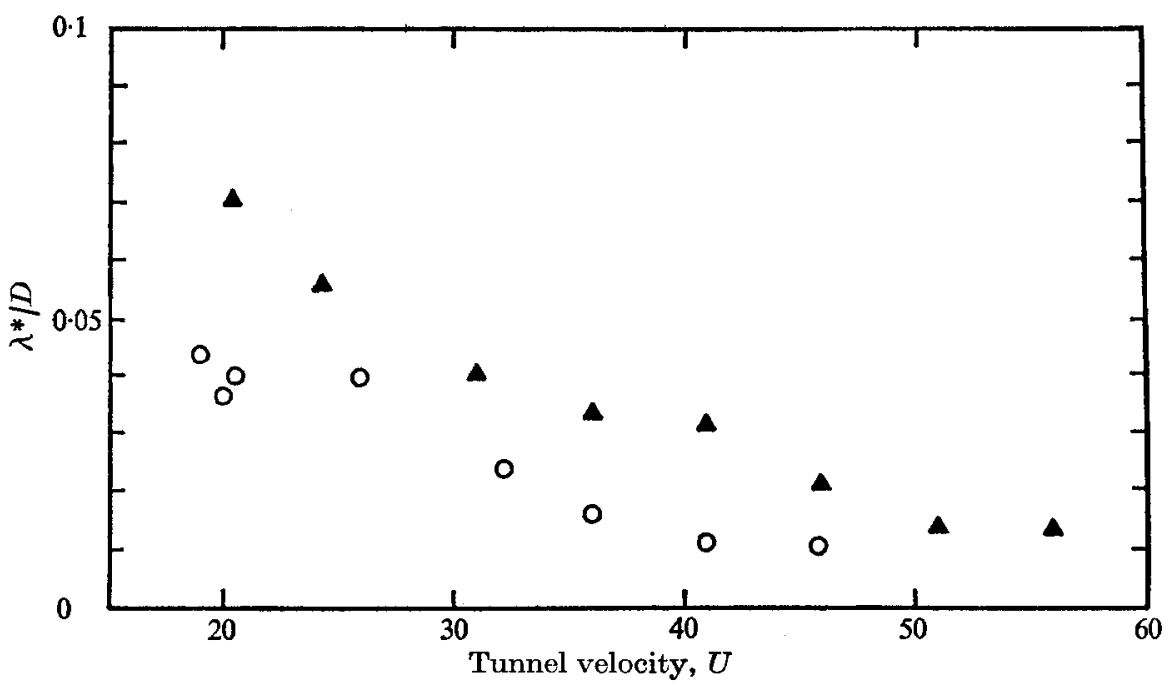

FIgure 14. Average value of the distance between two separations, over all meaningful cavitation numbers, against $U$, for hemispherical nose (O) and Swedish headform ( $\Delta$ ).

is independent of $\sigma$. Next an attempt will be made to generalize the above observations, based on physical arguments, and to obtain a correlation to predict the position of cavitation separation. This correlation will then be used to predict the point of cavitation separation on a sphere and a cylinder.

\section{A correlation for boundary-layer separation with cavitation}

From the results of $\$ 4$ it is clear that the original position of boundary-layer separation under non-cavitating conditions is shifted to a new upstream position owing to the presence of cavitation. In this section we consider determining this shift $X$, as a function of $\sigma$ and some relevant quantities existing under noncavitating conditions. $X$ is symbolically given by the difference

$$
\left[\left(S_{B} / D\right)_{\mathrm{NC}}-\left(S_{B} / D\right)_{\sigma}\right], \text { where }\left(S_{B} / D\right)_{\mathrm{NC}}
$$

is the position of boundary-layer separation existing under non-cavitating conditions, and $\left(S_{B} / D\right)_{\sigma}$ is the same quantity existing under cavitating conditions.

The extent of cavitation on the present two bodies was found to be determined by a normalized cavitation number $\sigma^{\prime}, \sigma^{\prime}=1+\sigma /\left(C_{s}\right)_{\mathrm{NC}}=\left(P_{s}-P_{v}\right) /\left(P_{s}-P_{t}\right)$, where $\left(C_{s}\right)_{\mathrm{NC}}$ is the pressure coefficient at the position of boundary-layer separation under non-cavitating conditions. The values of $\left(C_{s}\right)_{\mathrm{NC}}$ were determined with the help of the observed positions of boundary-layer separation and the pressure distribution measurements of Rouse \& McNown (1948), and Kermeen (1952) in the present tunnel facility. $\sigma^{\prime}$ is a useful parameter: it indicates the closeness of the pressure at laminar separation to the vapour pressure. When $\sigma^{\prime}$ was less than zero, no attached cavitation was ever observed. Attached cavitation was first observed when $\sigma^{\prime} \doteq 0$, and, as $\sigma^{\prime}$ was further increased, progressively 

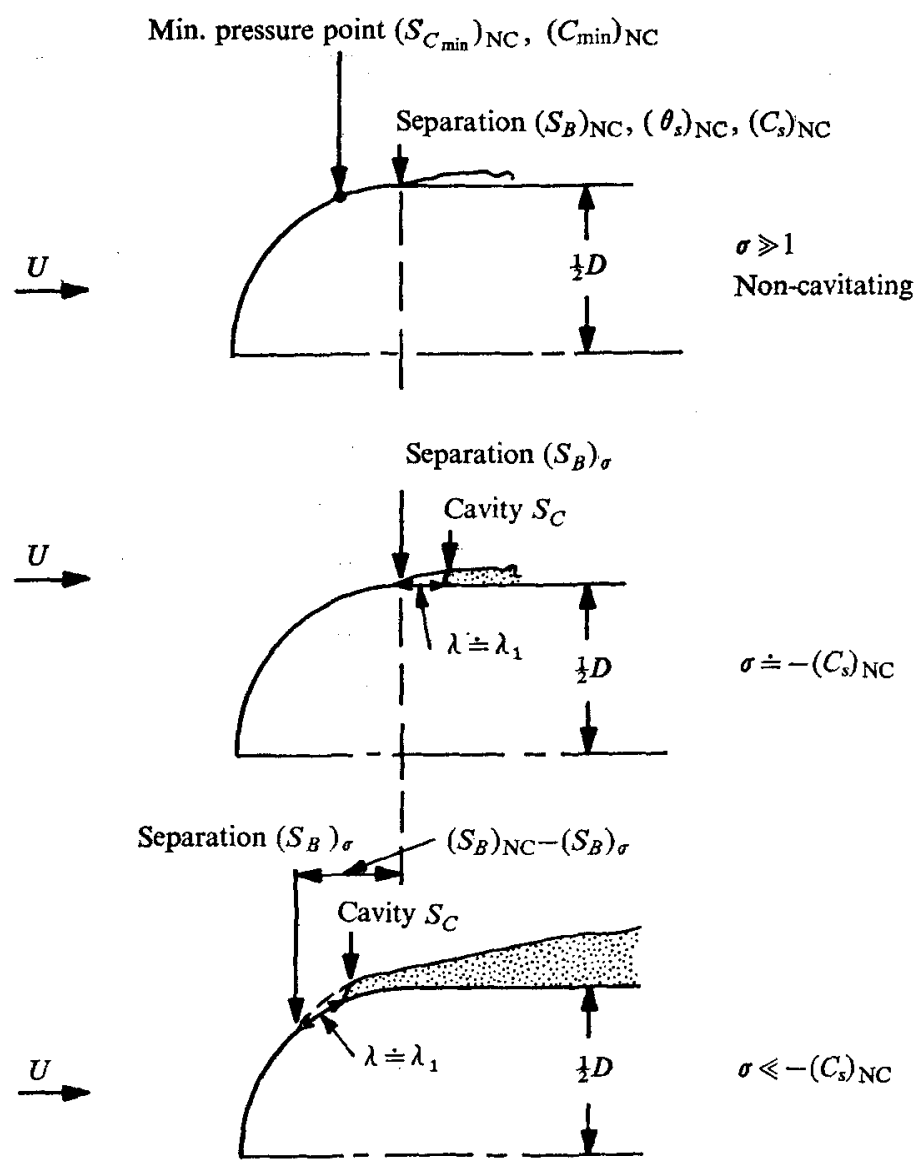

FrGURE 15. Diagram showing the development of cavitation with reduction of tunnel pressure, at a fixed velocity, on both test bodies.

more cavitation was seen. From this, it is expected that $X$ is at least dependent on $\sigma^{\prime}$. In addition, $X$ was found to be related to the length parameter

$$
\left[\left(S_{B} / D\right)_{\mathrm{NC}}-\left(S_{C_{\mathrm{min}}} / D\right)_{\mathrm{NC}}\right]
$$

$\left(S_{B} / D\right)_{\mathrm{NC}}$ is the position of boundary-layer separation under non-cavitating conditions and $\left(S_{C_{\min }} / D\right)_{\mathrm{NC}}$ is the streamwise position of the minimum pressure point under non-cavitating conditions. The value of $\left(S_{C_{\mathrm{min}}} / D\right)_{\mathrm{NC}}$ for our test bodies was obtained from Rouse \& MoNown (1948). The important parameters in determining the position of boundary-layer separation with cavitation are shown in the figure 15. The non-dimensional groups formed from the relevant parameters (already mentioned) were

$$
\sigma^{\prime}=\left[1+\frac{\sigma}{\left(C_{s}\right)_{\mathrm{NC}}}\right] \text { and } \Phi=\left[\frac{\left(S_{B} / D\right)_{\mathrm{NC}}-\left(S_{B} / D\right)_{\sigma}}{\left(S_{B} / D\right)_{\mathrm{NC}}-\left(S_{C_{\mathrm{m} \text { In }}} / D\right)_{\mathrm{NC}}}\right]
$$

A plot of $\sigma^{\prime}$ against $\Phi$ for our test bodies is shown in figure 16 and it may be said that the data collapse fairly well. Figure 16 indicates that certain quantities, known under non-cavitating conditions, are sufficient to determine the position of 


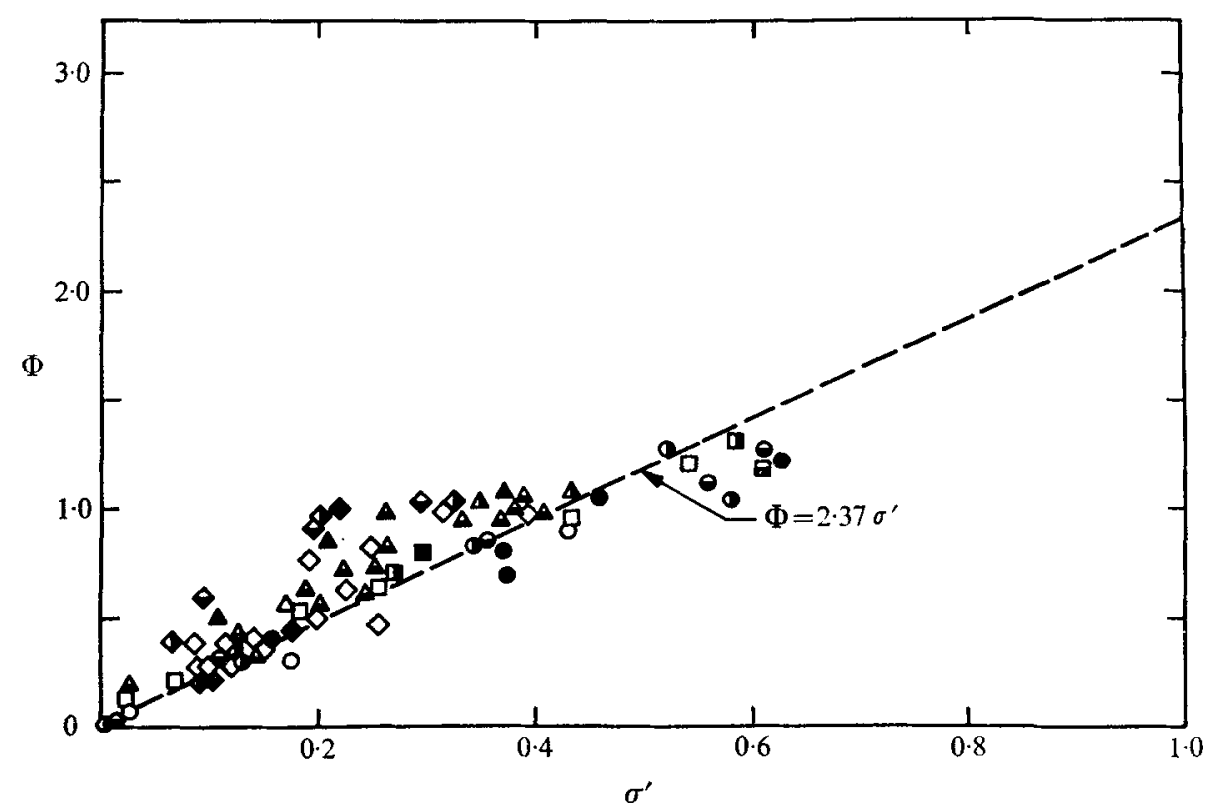

Frgure 16. Correlation plot, to find the position of boundary-layer separation under cavitating conditions.

$\begin{array}{lcccccccc}U\left(\mathrm{ft} \mathrm{s}^{-1}\right) & 20 & 25 & 30 & 35 & 40 & 45 & 50 & 55 \\ \text { Headform }(D=1.755 \text { in. }) & \triangle & \Delta & \Delta & \Delta & \diamond & \diamond & \ominus & \bullet \\ \text { Nose }(D=1.813 \text { in. }) & \bigcirc & \bullet & \ominus & \bullet & \square & \square & \square & \square\end{array}$

boundary-layer separation in the presence of cavitation as a function of cavitation number $\sigma$. A straight-line approximation to the data of figure 16 gives

$$
\Phi=2 \cdot 37 \sigma^{\prime}
$$

As will be seen, use of (1) for a range of $\sigma^{\prime}$ wider than that shown in figure 16 provided good agreement between predicted and observed positions of cavitation separation on sphere and cylinder.

\section{A correlation for the distance between the two separations}

The physical appearance of cavitation in present models, as represented by figure 1(b) (plate 1), has a striking similarity to that of cavitation (in the form of air entrainment) in the diverging sections of oil-film lubrication bearings, as illustrated by Taylor (1964). Although the two flow configurations are quite different, especially in terms of inertial effects, the similarity arises because cavitation in the present models occurs in the separated zone of the viscous laminar boundary layer, where inertial effects may be quite small as compared with inertial effects in the outer flow. Pearson (1960) conducted experiments similar to that of Taylor but with simpler apparatus. The apparatus consisted of a symmetric wedge with a divergence angle of $\frac{1}{20}$, placed over a flat plate with minimum clearance of $0.004 \mathrm{in}$.; and the region between the two was filled with different types of liquid with viscosity high compared with that of water. The 


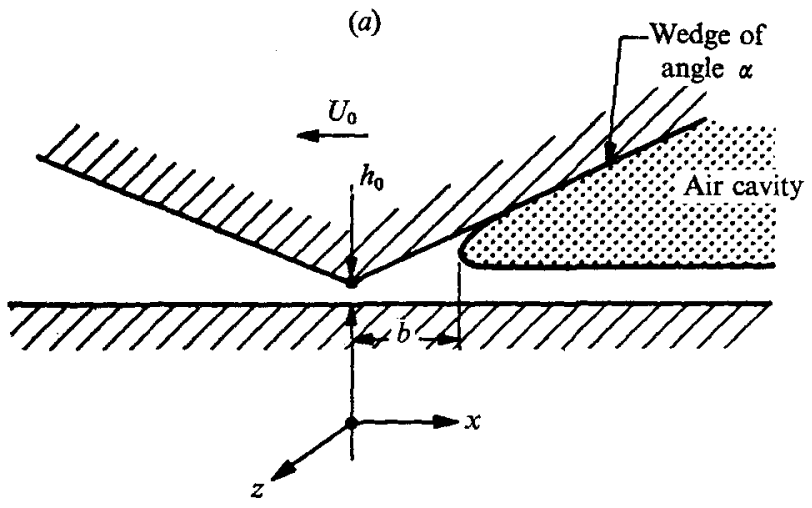

(b)

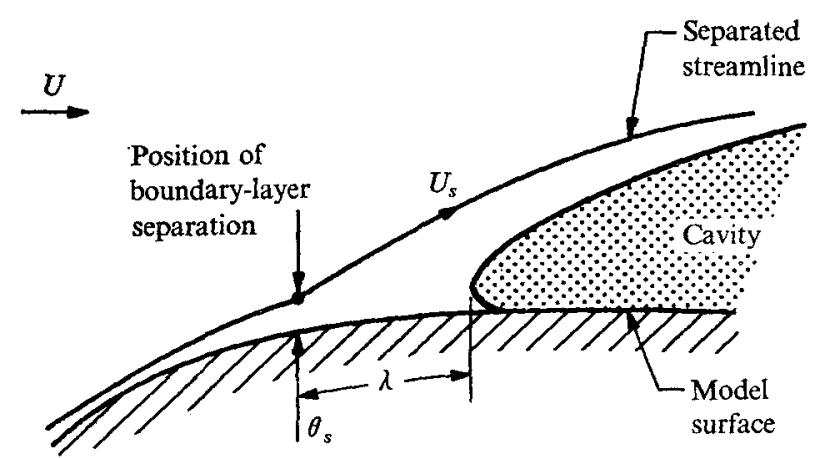

Fraure 17. Diagram showing the physical conditions of cavitation formation in (a) diverging section of a wedge (Pearson 1960), $(b)$ boundary-layer separated region of both test bodies.

symmetric wedge was terminated at $x=+1$ in. and $x=-1$ in., in the notation of figure $17(a)$. It was drawn at measured constant speed perpendicular to planes $x=$ constant. This resulted in the suction of an air cavity through the diverging section of the apparatus, and the position of the meniscus was determined with flash photographs. The air cavity possessed a characteristic instability in the $z$ direction, with alternating air fingers and fully wetted regions. Similar instability is noticed with cavitation on present bodies, as in figure $1(b)$ (plate 1 ). In the experiments of Taylor and Pearson, the important dimensionless group was found to be $\mu U_{0} / T$ ( $\mu$ is the coefficient of dynamic viscosity, $T$ is the coefficient of surface tension), with the minimum clearance $h_{0}$ as the pertinent length parameter. $\mu U_{0} / T$ is commonly known as the Taylor-Saffman parameter; it is the ratio of Weber number to Reynolds number, both based on $U_{0}$. The physical conditions under which cavitation occurs in the present models are shown in figure $17(b)$; and the likeness to conditions shown in figure $17(a)$ is very suggestive. With this in mind, the important dimensionless group was taken to be the Taylor-Saffman parameter, with Weber number based on local velocity at boundary-layer separation, and Reynolds number based on free stream velocity. The length parameter equivalent to $h_{0}$ was chosen to be the boundary-layer momentum thickness 


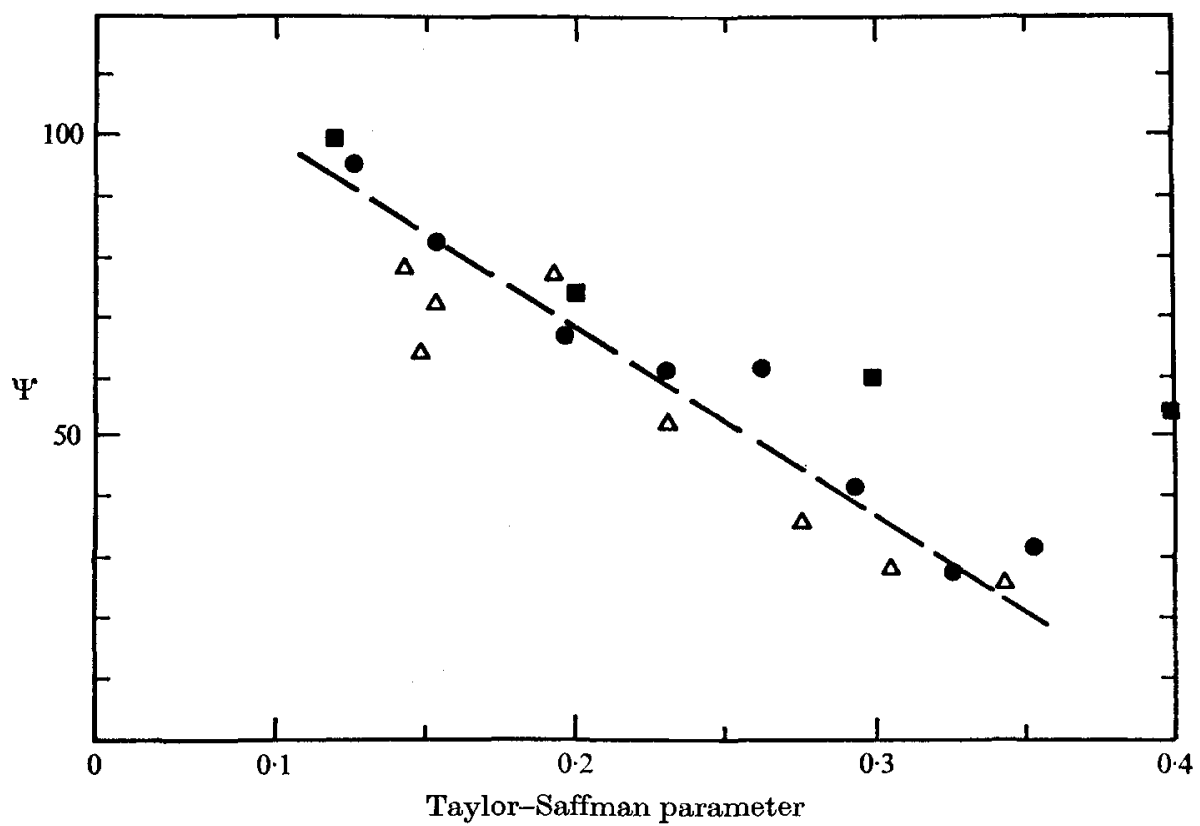

Figure 18. Correlation plot, to find the distance from the position of boundary-layer separation to that of cavitation separation. - Swedish hoadform, $\Psi=\lambda^{*} /\left(\theta_{s}\right)_{\mathrm{NC}} ; \Delta$, hemispherical nose, $\Psi^{*}=\lambda^{*} /\left(\theta_{s}\right)_{\mathrm{NC}} ; \square$, Pearson's data, $\Psi^{*}=b / h_{0}$.

at separation $\theta_{s}$. As discussed earlier, $\lambda$ was independent of $\sigma$; and this allows us to choose the pertinent variables in determining $\lambda$ at any cavitation number. The most useful condition to choose is that under non-cavitating conditions, or for $\sigma \gg 1$. Thus the non-dimensional groups chosen were

$$
\lambda * /\left(\theta_{s}\right)_{\mathrm{NC}} \text { and }(\mu U / T)\left[1-\left(C_{s}\right)_{\mathrm{NC}}\right] \text {. }
$$

A plot of these quantities for the present models is shown in figure 18. The values of $\left(\theta_{s}\right)_{\mathrm{NC}}$ were computed by Thwaites's approximate method (Arakeri 1973). Also included in figure 18 are results obtained by Pearson; $b / h_{0}$ in Pearson's experiments is equivalent to $\lambda^{*} /\left(\theta_{s}\right)_{\mathrm{NC}}$ for the present experiments and $\mu U_{0} / T$ is equivalent to $(\mu U / T)\left[1-\left(C_{s}\right)_{\mathrm{NC}}\right]$. The agreement between our results and those of Pearson is quite good, especially for low values of the Taylor-Saffman parameter. In our analysis, the possible role of another geometrical parameter, equivalent to the wedge angle in Pearson's experiments, is not considered. In our problem, this parameter would be the angle between the viscous separated streamline and the adjacent boundary of the solid body. Neglect of it may be responsible for some of the differences in the observations of figure 18 .

A straight-line approximation to the data for our test bodies, in figure 18, gives

$$
\frac{\lambda^{*}}{\left(\theta_{\mathrm{s}}\right)_{\mathrm{NC}}}=130-312 \frac{\mu U}{T}\left[1-\left(C_{s}\right)_{\mathrm{NC}}\right]
$$

Since $\lambda^{*}$ cannot be negative, it should be taken that

$$
\frac{\lambda^{*}}{\left(\theta_{s}\right)_{\mathrm{NC}}}=0 \text { for } \frac{\mu T}{T}\left[1-\left(C_{s}\right)_{\mathrm{NC}}\right]>0 \cdot 42 \text {. }
$$




\section{The complete correlation}

One can express the position of cavitation separation as the sum of the position of the boundary-layer separation preceding it and the distance between the two, or

$$
\left(\frac{S_{C}}{D}\right)=\left(\frac{S_{B}}{D}\right)+\left(\frac{\lambda^{*}}{D}\right)
$$

Using the correlations obtained in (1) and (2), (3) may be written as

$$
\begin{aligned}
&\left(\frac{S_{C}}{D}\right)=\left(\frac{S_{B}}{D}\right)_{\mathrm{NC}}-2 \cdot 37[1+\left.\frac{\sigma}{\left(C_{s}\right)_{\mathrm{NC}}}\right] \times\left[\left(\frac{S_{B}}{D}\right)_{\mathrm{NC}}-\left(\frac{S_{C_{\min }}}{D}\right)_{\mathrm{NC}}\right] \\
&+\left(\frac{\theta_{s}}{D}\right)_{\mathrm{NC}}\left\{130-312 \frac{\mu U}{T}\left[1-\left(C_{s}\right)_{\mathrm{NC}}\right]\right\}
\end{aligned}
$$

To use (4), knowledge of the pressure distribution on the cavitating body under non-cavitating conditions, but at the same Reynolds number, is required. Similarly, the position of boundary-layer separation under non-cavitating conditions should be known experimentally, or may be estimated by Thwaites's approximate method. For our test bodies, Thwaites's method predicted the position of laminar boundary-layer separation to within $2 \%$ of that observed (Arakeri 1973). Again, Thwaites's method may be used to compute $\theta_{s}$ under non-cavitating conditions. Equation (4) is valid only for

$$
(\mu U / T)\left[1-\left(C_{s}\right)_{\mathrm{NC}}\right]<0 \cdot 42
$$

for values of this term greater than $0 \cdot 42$, the third term representing $\left(\lambda^{*} / D\right)$ is zero.

\section{Computations using the complete correlation}

Computations were made to predict the position of cavitation separation on spheres and cylinders for various Reynolds and cavitation numbers. For these, it is convenient to express (4) in terms of angular position:

$$
\begin{aligned}
\gamma_{C}=\left(\gamma_{B}\right)_{\mathrm{NC}}-2 \cdot 37 & {\left[1+\frac{\sigma}{\left(C_{s}\right)_{\mathrm{NC}}}\right] \times\left[\left(\gamma_{B}\right)_{\mathrm{NC}}-\left(\gamma_{C \min }\right)_{\mathrm{NC}}\right] } \\
& +\left(\frac{360}{3 \cdot 14}\right)\left[\left(\frac{\theta_{s}}{D}\right)_{\mathrm{NC}}\right]\left\{130-312 \frac{\mu U}{T}\left[1-\left(C_{s}\right)_{\mathrm{NC}}\right]\right\} .
\end{aligned}
$$

$\gamma$ represents the angular position from the stagnation point, in degrees. The different flow regimes possible on spheres and cylinders are as first defined by Roshko (1961). The first critical Reynolds number is when the separated laminar free shear layer re-attaches, to form a laminar separation bubble. Therefore, for $R$ less than first critical Reynolds number, only laminar separation exists; and, for $R$ greater than first critical Reynolds number, both laminar and turbulent separations exist. The following information under non-cavitating conditions was obtained with the help of experimental pressure distribution measurements of Maxworthy (1969) on spheres and Roshko (1961) on cylinders. 


\section{Spheres}

(i) $10^{4}<R<10^{5}$ : below the first critical Reynolds number,

Thwaites's method,

$$
\left(\gamma_{B}\right)_{\mathrm{NC}}=82 \cdot 5^{\circ}, \quad\left(\gamma_{C_{\min }}\right)_{\mathrm{NC}} \doteq 74^{\circ}
$$

$$
\left(C_{s}\right)_{\mathrm{NC}} \doteq-0 \cdot 4, \quad\left(\theta_{s} / D\right)_{\mathrm{NC}} \doteq 0 \cdot 284 / R^{\frac{1}{2}} .
$$

(ii) $R=2 \times 10^{5}$ : at the first critical Reynolds number,

$$
\begin{aligned}
& \left(\gamma_{B}\right)_{\mathrm{NC}}=82 \cdot 5^{\circ}, \quad\left(\gamma_{C_{\mathrm{min}}}\right)_{\mathrm{NC}} \doteq 72 \cdot 5^{\circ} \\
& \left(C_{s}\right)_{\mathrm{NC}} \doteq-0 \cdot 46, \quad\left(\theta_{\mathrm{s}} / D\right)_{\mathrm{NC}} \doteq 6 \times 10^{-4} .
\end{aligned}
$$

(iii) $3 \times 10^{5}<R<10^{6}$ : above the first critical Reynolds number,

$$
\begin{gathered}
\left(\gamma_{B}\right)_{\mathrm{NC}}=109^{\circ}, \quad\left(\gamma_{o_{\mathrm{m} 1 \mathrm{~N}}}\right)_{\mathrm{NC}}=90^{\circ} \\
\left(C_{s}\right)_{\mathrm{NC}}=-1.02, \quad\left(\theta_{s} / D\right)_{\mathrm{NC}}=0 \cdot 4 / R^{\frac{1}{2}}
\end{gathered}
$$

\section{Cylinders}

(i) $10^{4}<R<10^{5}$ : below the first critical Reynolds number,

$$
\begin{gathered}
\left(\gamma_{B}\right)_{\mathrm{NC}} \doteq 80^{\circ}, \quad\left(\gamma_{C_{\mathrm{min}}}\right)_{\mathrm{NC}} \doteq 70^{\circ} ; \\
\left(C_{s}\right)_{\mathrm{NC}} \doteq-1.1875, \quad\left(\theta_{s} / D\right)_{\mathrm{NO}} \doteq 0.32 / R^{2}
\end{gathered}
$$

The momentum thickness at boundary-layer separation under non-cavitating conditions was estimated by Thwaites's method at the predicted position of laminar separation. The predicted positions were the same, to within $7 \%$, as those observed experimentally.

$$
\begin{gathered}
\text { Sample calculations: spheres } \\
U=10 \mathrm{ft} \mathrm{s}^{-1}, \quad \sigma=0 \cdot 1, \quad D=\frac{1}{2} \text { in. } \\
R=4 \times 10^{4} \Rightarrow\left(\theta_{s} / D\right)_{\mathrm{NC}}=0.284 /\left(4 \times 10^{4}\right)^{\frac{1}{2}}=14 \cdot 2 \times 10^{-4} .
\end{gathered}
$$

As mentioned,

Therefore,

$$
\left(\gamma_{B}\right)_{\mathrm{NC}} \doteq 82 \cdot 5^{\circ}, \quad\left(\gamma_{C_{\mathrm{min}}}\right)_{\mathrm{NC}} \doteq 74^{\circ}, \quad\left(C_{s}\right)_{\mathrm{NC}} \doteq-0 \cdot 4
$$

$$
\frac{\mu U}{T}\left[1-\left(C_{s}\right)_{\mathrm{NC}}\right]=0 \cdot 061, \quad\left[1+\frac{\sigma}{\left(C_{s}\right)_{\mathrm{NC}}}\right]=0 \cdot 75
$$

Using these values in (5),

$$
\begin{aligned}
& \gamma_{C}=82 \cdot 5^{\circ}-[2 \cdot 37(0 \cdot 75)\left.\left(82 \cdot 5^{\circ}-74^{\circ}\right)\right]+\left\{360 / 3 \cdot 14 \times 14 \cdot 2 \times 10^{-4}\right. \\
&\times[130-312(0 \cdot 061)]\}^{\circ}=82 \cdot 5^{\circ}-15 \cdot 1^{\circ}+18 \cdot 1^{\circ}=85 \cdot 5^{\circ} .
\end{aligned}
$$

Similar calculations for spheres and cylinders, at various Reynolds and cavitation numbers, are shown in figures $19(a)-(c)$. 


\section{H. Arakeri}
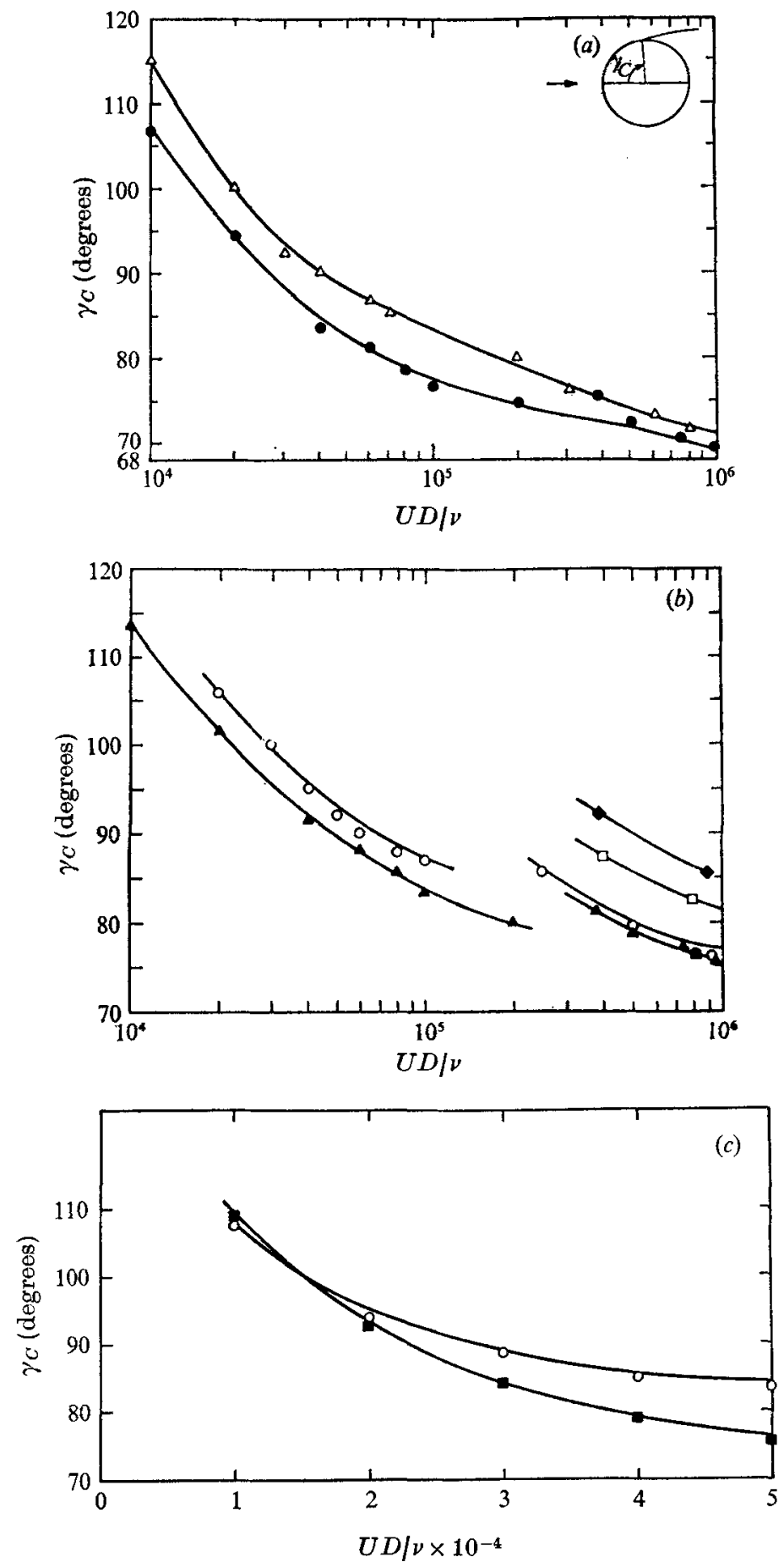

FIGURE 19. Comparison of calculated with observed position of cavitation separation. $(a)$, (b) 0.25 and $0.5 \mathrm{in}$. sphere; (c) $0.25 \mathrm{in}$. cylinder. $\gamma_{c}$ calculated from the smooth-separation condition by Brennen.

(a)

$0 \cdot 1$

Observed (Brennen)

Calculated (present)

$\gamma_{c}$ (degrees) (b)

(c)

$\begin{array}{cc}0.25 & 0.5 \\ \bigcirc & \square \\ 60 & \end{array}$




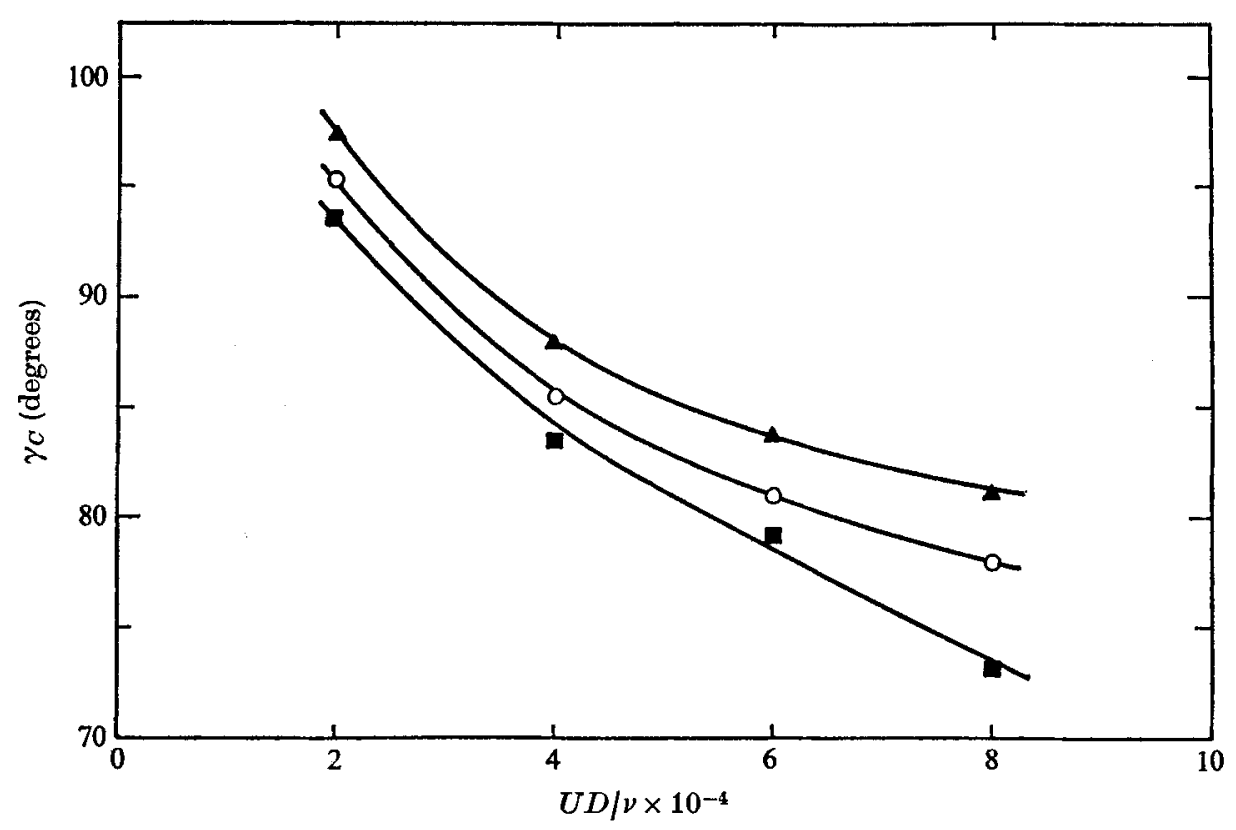

Figure 20. Calculated position of cavitation separation on $0.5 \mathrm{in}$. sphere, at $\sigma=0 \cdot 1$.

$$
\begin{array}{cccc} 
& \text { Mercury } & \text { Water } & \text { Benzene } \\
\mu / T\left(\times 10^{3} \mathrm{~s} \mathrm{ft}^{-1}\right) & 0.9 & 4.36 & 6.85 \\
& \Delta & \bigcirc &
\end{array}
$$

\section{Comparison with experiments}

Also shown in figures $19(a)-(c)$ are positions of cavitation separation found experimentally by Brennen $(1969 a, 1970)$. Marked, too, is the position of cavitation obtained theoretically, using the smooth-separation condition (Brennen $1969 b$ ). Since these are based on potential flow calculations, the predicted positions would not be dependent on Reynolds number.

From figure $19(a)$, at $\sigma=0 \cdot 1$ the calculated values follow the same trend, with $R$, as the experimentally observed values, to within $7 \%$. From figure $19(b)$, at $\sigma=0.25$ and 0.5 the agreement is much better; the calculated values are the same to within $4 \%$ as those observed. The important point is that the slope of the calculated and experimentally found curves is in excellent agreement for all Reynolds and cavitation numbers. From figure $19(b)$, at $\sigma=0 \cdot 25$ the discontinuity in the observed positions, just before and after the first critical Reynolds number for a sphere, is also predicted from our calculations. From figure $19(c)$, the case of a cylinder, agreement is very good at $R=10^{4}$, but gradually differs by about $10 \%$ at $R=10^{5}$.

Predictions of the position of cavitation separation for three different fluids, which have different $\mu / T$, are shown in figure 20 . The value of the coefficient of surface tension $T$ of mercury is about eight times that of water; and for benzene it is one-half that of water. From figure 20, for the low Reynolds number range 
$R<6 \times 10^{4}$, viscous forces dominate in determining the position of cavitation separation on a sphere; and this is consistent with the observations of Brennen (1970). However, at higher Reynolds numbers, surface tension is seen to influence the position of cavitation separation. This is in contrast to Brennen's observations, which show that, even up to $R=1 \cdot 2 \times 10^{5}$, surface tension does not affect the position of cavitation separation.

I should like to express my sincere appreciation to Dr A. J. Acosta, under whose supervision this work was carried out, and formed part of a D.Phil. thesis at the California Institute of Technology. This research was partly carried out under the Naval Ship Systems Command General Hydromechanics Research Program Subproject SR 02301 01, administered by the Naval Ship Research and Development Centre, under Office of Naval Research contract N00014-67-A-0094-0023. A generous grant from the Sloan Foundation is also greatly appreciated. The author wishes to thank the CIT Hydro Aeronautics Laboratory staff for their assistance in the experimental work, and Dr C. Brennen for helpful discussions on the subject. The author also wishes to thank the referee for his helpful comments and criticism on the original manuscript.

\section{REFERENCES}

Acosta, A. J. \& Hamaguchi, H. 1967 Cavitation inception on the ITTC standard headform. Cal. Inst. Tech. Rep. E-149-1.

Arakeri, V.H. 1971 Water tunnel investigation of scale effects in cavitation detachment from smooth slender bodies and characteristics of flow past a bi-convex hydrofoil. Cal. Inst. Tech. Rep. E-79A-12.

ArakerI, V.H. 1973 Viscous effects in inception and development of cavitation on axisymmetric bodies. Cal. Inst. Tech. Rep. E-183-1.

Armstrong, A. H. 1953 Abrupt and smooth separation in plane and axisymmetric flow. Mem. Arm. Res. Est. G.B. no. 22/63.

Bland, R. E. \& Pelick, T. J. 1962 The schlieren method applied to flow visualization in a water tunnel. J. Basic Engng, A.S.M.E. 84, 587-592.

Brennen, C. 1969 a Some viscous and other real fluid effects in fully developed cavity flows. Cavitation: State of Knowledge. A.S.M.E.

Brennen, C. $1969 b$ A numerical solution of axisymmetric cavity flows. J. Fluid Mech. Mech. 37, 671.

Brennes, C. 1970 Some cavitation experiments with dilute polymer solutions. J. Fluid Mech. 44, 51 .

GASTER, M. 1967 The structure and behaviour of laminar separation bubbles. NPL Aero. Rep. no. 1181 (revised).

HoyT, J. W. 1966 Wall effect on ITTC standard head shape pressure coefficients. 11th ITTC Formal Contribution.

KERMeEN, R. W. 1952 Some observations of cavitation on hemispherical head models. Cal. Inst. Tech. Rep. E-35-1.

KNaPP, R. T., Levy, J., O'NeILL, J. P. \& Brown, F. B. 1948 The Hydrodynamics Laboratory of the California Institute of Technology. Trans. A.S.M.E. 20, 437.

Maxworthy, T. 1969 Experiments on the flow around a sphere at high Reynolds numbers. J. Appl. Mech. A.S.M.E. 36, 1.

Parkin, B. R. \& Kermeen, R. W. 1953 Incipient cavitation and boundary layer interaction on a streamlined body. Cal. Inst. Tech. Hydrodynamics Lab. Rep. E-33-2. 
Pearson, J. R. A. 1960 The instability of uniform viscous flow under rollers and spreaders. J. Fluid Mech. 7, 481.

Plesset, M. S. 1949 The dynamies of cavitation bubbles. J. Appl. Mech. A.S.M.E. 16, 277.

Rosнko, A. 1961 Experiments on a cylinder at high Reynolds numbers. J. Fluid Mech. 10,345 .

Rotse, H. \& McNown, J. S. 1948 Cavitation and pressure distribution headforms at zero angle of yaw. State University of Iowa, Eng. Bull. no. 32.

TAYLOR, G. I. 1964 Cavitation in hydrodynamic lubrication. Cavitation in Real Liquids. Elsevier.

Thwartes, B. 1949 Approximate calculation of laminar boundary layer. Aero. Quart. 1, 24.5.

Wu, T. Y. 1968 Inviscid cavity and wake flows. Basic Developments in Fluid Dynamics. Academic. 